\title{
Tuberculosis en cabeza y cuello. Experiencia en la Patagonia chilena y revisión de la literatura
}

\author{
Tuberculosis in head and neck. Experience in Chilean Patagonia and \\ literature review
}

\author{
Carlos Morales A. ${ }^{1,2}$, Daniela Gallardo G. ${ }^{3}$, Camilo Iñiguez L. ${ }^{1}$
}

\begin{abstract}
Resumen
La tuberculosis (TBC) es una de las patologías infecciosas que más muertes ha producido a nivel mundial, sobrepasando al virus de inmunodeficiencia humana (VIH). En Chile a pesar de la baja incidencia de la patología, aun no es posible llegar a su erradicación. La TBC es una enfermedad que habitualmente compromete el pulmón, sin embargo, en ocasiones compromete otros sitios del organismo, siendo la ubicación en cabeza y cuello una de las más importantes. Las manifestaciones clínicas son variadas y el curso de estas puede ser larvado, simulando otras patologías de mayor frecuencia en el ámbito otorrinolaringológico y transformando su diagnóstico en un desafío mayor. En este artículo se presentan tres casos clínicos diagnosticados y manejados en nuestra en región, además de una revisión de la literatura disponible en relación a la presentación clínica, orientación diagnóstica y tratamiento de la patología.

Palabras clave: Tuberculosis, cabeza y cuello, adenopatía.
\end{abstract}

\begin{abstract}
Tuberculosis is one of the infectious diseases which has produced more deaths around the world, even more than human immunodeficiency virus. In Chile, despite of the low incidence, there is still not possible to eradicate it. Tuberculosis is a disease which commonly compromises lungs, however, in some occasions involves another site of the organism, been the location in head and neck one of the most important. The clinical features are diverse and the natural history of those can be masked, for this reason it can simulate another more frequent disease in otolaryngology field, making the diagnosis a real challenge. In this article, we present three clinical cases diagnosed and treated in our region, besides, a review of the available literature related with the clinical presentation, diagnosis orientation and treatment of this pathology.
\end{abstract}

Keywords: tuberculosis, head and neck, adenopathy.

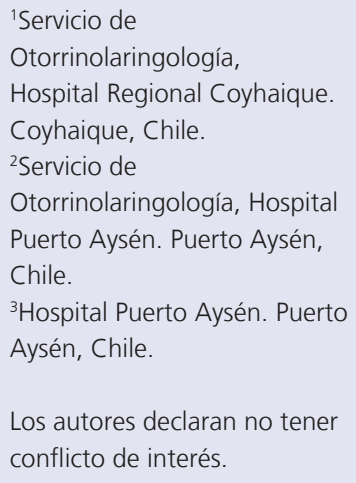

Recibido el 4 de noviembre de 2020. Aceptado el 16 de marzo de 2021.

Correspondencia: Carlos Morales A. Jorge Ibar 068, Coyhaique. Hospital Regional Coyhaique, XI región. Coyhaique, Chile. Email: dr.cmorales.a@gmail. com

\section{Introducción}

La tuberculosis (TBC) es una de las patologías infecciosas que más muertes ha producido a nivel mundial, sobrepasando al virus de inmunodeficiencia humana (VIH). La incidencia de TBC disminuyó con la aparición de la vacuna BCG, sin embargo, ha aumentado progresivamente dado la pandemia de VIH y la resistencia a los antibióticos utilizados para el tratamiento de la TBC pulmonar. De acuerdo con los datos de la OMS, los pacientes con $\mathrm{TBC}$ en cualquiera de sus presentaciones clínicas han llegado a 10,4 millones de nuevos casos y 1,8 millones de muertes en el mundo en el año $2015^{1}$.

Aun cuando, en Chile tenemos buenos resultados en cuanto a la disminución de la morbimortalidad en las últimas décadas, lo cual nos ha posicionado como uno de los países con tasas más bajas dentro de la región, hemos visto estancada la incidencia en valores cercanos a 13 casos por 100.000 habitantes, en los últimos 5 años según el pasado reporte de si- 
tuación del Ministerio de Salud en el año 2014, mostrando pocos avances en relación con lo que se esperaría de un país que está siendo exitoso en la erradicación de la enfermedad ${ }^{2}$.

La infección por TBC generalmente se manifiesta a nivel pulmonar, sin embargo, también puede afectar otros sitios en el organismo, lo cual se conoce como TBC extrapulmonar (TBEP). La TBEP corresponde al 15\% de los casos nuevos de TBC, y de estos el 10\% a $35 \%$ se manifiesta en cabeza y cuello. Las manifestaciones en cabeza y cuello pueden presentarse en diversos sitios: región cervical, laringe, orofaringe, senos paranasales, rinofaringe, oídos, glándulas salivales ${ }^{3}$. Sin embargo, el sitio más común de presentación es a nivel del cuello como una linfoadenopatía crónica, también conocida como escrófula ${ }^{4}$.

El diagnóstico de la enfermedad es un desafío dado las múltiples presentaciones clínicas de esta, dependiendo del sitio de infección, simulando en ocasiones una neoplasia de la vía aereodigestiva superior con diseminación locorregional a nivel del cuello, una neoplasia laríngea avanzada con obstrucción de la vía aérea, una infección larvada a nivel del oído o de las glándulas salivales que no responde de forma habitual al tratamiento ${ }^{5}$.

El gold standard para el diagnóstico es el cultivo de secreción o tejido, obtenido de punción aspirativa de una masa cervical o biopsia a través de cirugía, ya que en TBEP la sensibilidad de estudios de esputo tiene una sensibilidad y especificidad baja ${ }^{6}$. Sin embargo, en la actualidad existen métodos de amplificación de ADN que han mejorado el estudio diagnóstico en algunos centros. En Chile, el tratamiento y seguimiento de los pacientes está a cargo de la atención primaria de salud y el tratamiento farmacológico como tal, corresponde a una terapia asociada de antibióticos con duración total de 6 meses $^{7}$.

El siguiente estudio muestra una serie de casos presentados en los últimos dos años en la Región de Aysén, con distintas presentaciones clínicas que han motivado la revisión de la literatura en relación con esta patología.

\section{Caso Clínico 1}

Paciente de sexo masculino, 54 años, con antecedentes mórbidos de tabaquismo e hi- pertensión arterial, antecedente social de estar privado de libertad, ingresado a Servicio de Urgencia de Hospital Regional de Coyhaique por distrés respiratorio agudo que requiere de intubación orotraqueal pesquisando importante masa laríngea que obstruye vía aérea. Se realizó traqueostomía y posteriormente laringoscopia directa con toma de biopsia para confirmación diagnóstica, con alta sospecha de tumor laríngeo avanzado. Resultado biopsia: mucosa con inflamación crónica, cambios epiteliales reactivos y desarrollo de granulomas necrotizantes de tipo tuberculoideo, sin células neoplásicas. Se confirma con baciloscopias positivas y se inicia tratamiento anti TBC. Al mes de inicio de tratamiento, se retira cánula de traqueostomía con indemnidad laríngea (Figura 1).

\section{Caso Clínico 2}

Paciente sexo femenino, 34 años, sin patologías crónicas conocidas, consulta por cuadro de dos años de evolución, con adenopatías cervicales izquierdas de crecimiento lento e indoloras, con episodios recurrentes de supuración con fístula crónica a piel. Se realiza tomografía computada de cuello donde se aprecia adenopatías cervicales y supraclaviculares izquierdas, así como submentonianas, asociadas a lesiones quísticas en relación con la parótida izquierda, una de ellas con trayecto fistuloso (Figura 2).

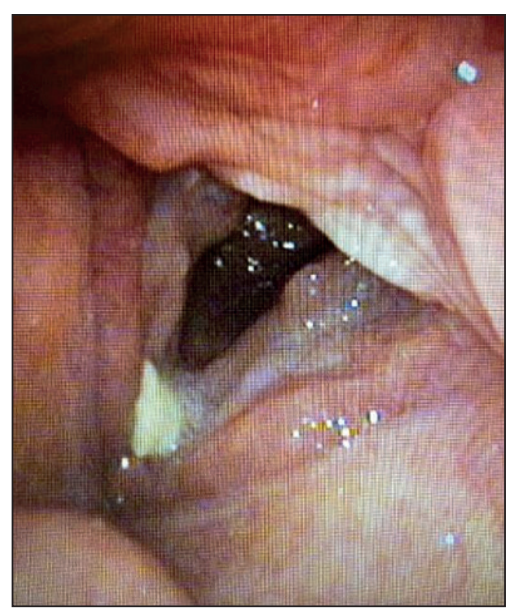

Figura 1. Laringe postratamiento anti-TBC. 


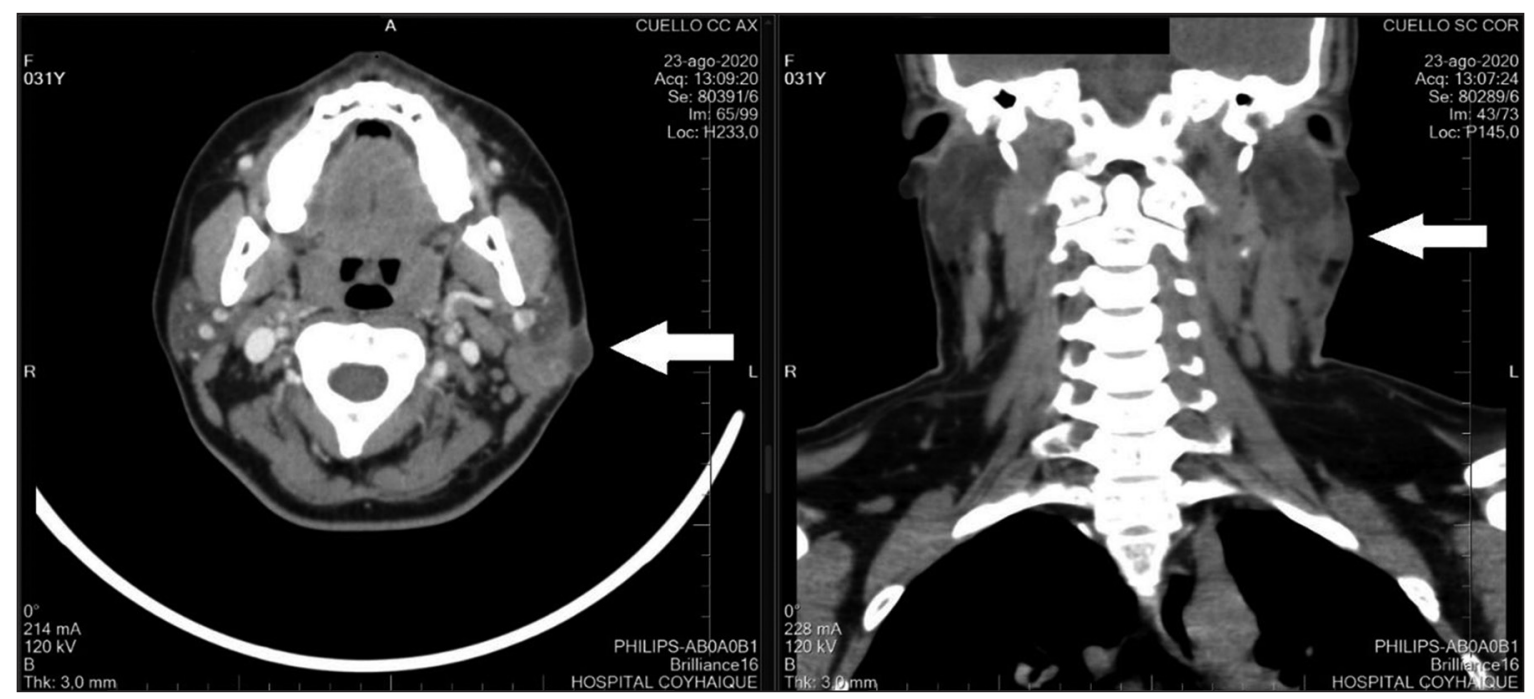

Figura 2. Tomografía de cuello, corte axial evidencia conglomerado de adenopatías a izquierda (flechas).

Se decide realizar biopsia excisional de lesión parotídea y del trayecto fistuloso, además de muestra de contenido quístico con confirmación positiva con método de amplificación de ácido nucleicos (Gene Xpert-RIF). Se recibe resultado de estudio anatomopatológico: fragmentos de glándula salival serosa con extensa inflamación crónica, granulomatosa, necrotizante de tipo tuberculoideo. Se inicia tratamiento estándar para TBC, con remisión de adenopatías cervicales.

\section{Caso Clínico 3}

Paciente sexo masculino, 39 años, derivado por cuadro de 3 meses de evolución caracterizado por tos, malestar general, sudoración nocturna, sensación febril y masa cervical izquierda de aproximadamente $5 \mathrm{~cm}$ de diámetro mayor, de consistencia pétrea, no dolorosa y sin cambios cutáneos. Estudio imagenológico con tomografía evidencia: nódulos de centro necrótico sugerente de adenopatías cavitadas en triángulo cervical anterior, específicamente grupo II izquierdo, en su conjunto miden 3,7 $\times 2,2 \mathrm{~cm}$, sugerentes de infección crónica por micobacterias (Figura 3 ). Se realiza estudio con baciloscopia la que resulta negativa, pero con cultivos positivos a los 30 días. Por lo que no se realiza otro estudio invasivo de la masa cervical y se inicia el tratamiento antituberculoso en atención primaria con buen resultado posterapia.

\section{Discusión}

Situación tuberculosis en Chile y en Región de Aysén

La región de Aysén cuenta con una superficie de $108.494 \mathrm{~km}^{2}$ y una población de 103.158 habitantes según el censo de 2017. Administrativamente está compuesta por cuatro provincias (Aysén, Capitán Prat, Coyhaique y General Carrera), las que están divididas en 10 comunas. Su capital regional es la ciudad de Coyhaique, la que cuenta con una población rural importante con una marcada lejanía entre las comunas y dificultades climáticas que destacan en época invernal. Además, la carencia de poseer una conexión totalmente terrestre del resto del país crea aún más un fenómeno de aislamiento del resto del territorio nacional.

A nivel país, la tasa de mortalidad por tuberculosis para el año 2012 fue de 1,5 por 100.000 , aumentando en 0,2 puntos respecto al año 2011. Esta cifra corresponde a 268 personas fallecidas por tuberculosis en el país durante el año $2012^{2}$.

La Figura 4 muestra la tasa de incidencia para TBC por región en Chile para el año 


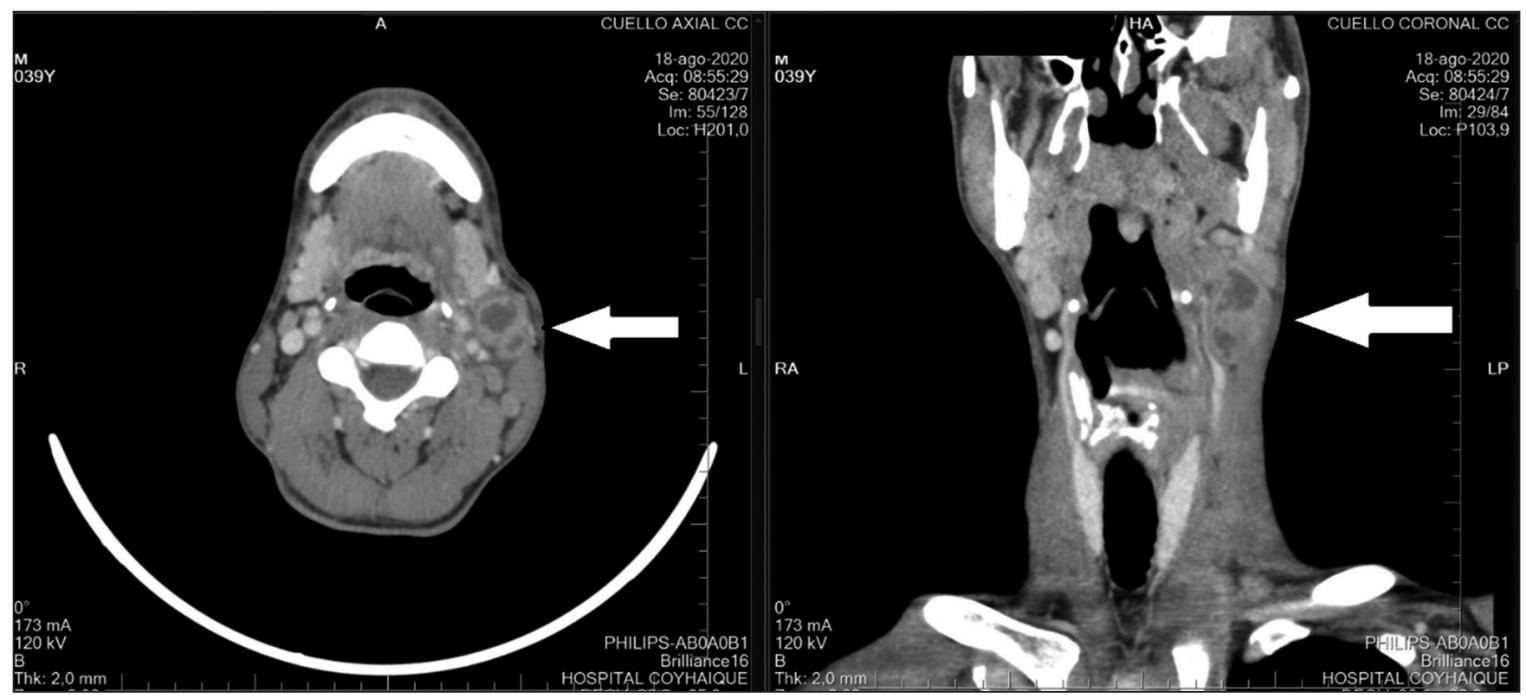

Figura 3. Tomografía de cuello, corte axial muestra compromiso intraparotídeo a izquierda (flechas).

Figura 4. Tabla incidencia de TBC, por región en el año 2014, fuente DEIS ${ }^{8}$.

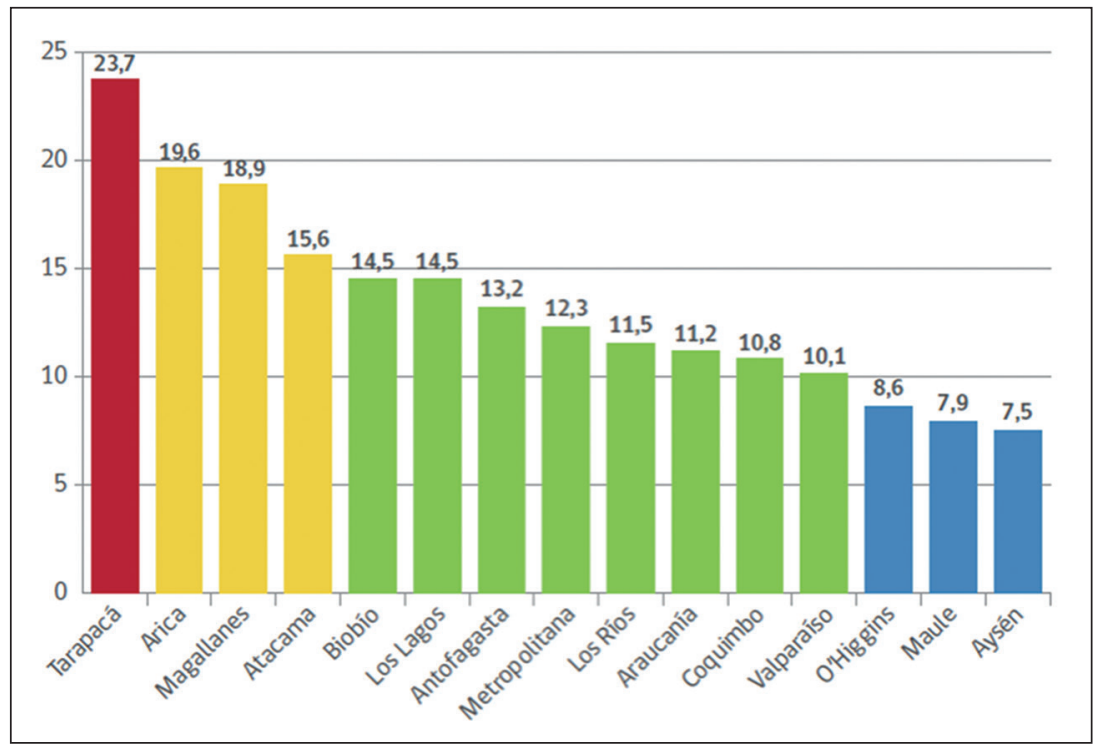

2014, sin hacer diferencia en pulmonar o extrapulmonar. Se observa que solamente la región de Tarapacá se mantiene sobre el umbral de eliminación de la enfermedad, con una tasa de 23,7 por 100.000 habitantes. Arica y Parinacota, Magallanes y Atacama tienen tasas consideradas muy altas, y solo O’Higgins, Maule y la Región de Aysén presentan tasas menores a 10 casos por 100.000 habitantes ${ }^{8}$.
Existen grupos dentro de la comunidad que han mostrado una mayor prevalencia de TBC. La Tabla 1 muestra la proporción de casos de TBC que pertenecen a alguno de los principales grupos de riesgo para enfermar de tuberculosis, según lo reportado por los Servicios de Salud a través del Registro Nacional en el año 2014. Para ese año el 37,2\% del total de casos de tuberculosis pertenece a alguno de los grupos de riesgo identificados. 


\section{Presentación clínica}

Las manifestaciones clínicas son variables y dependen del sitio de infección, del estado de la enfermedad y de la indemnidad del sistema inmune del paciente. La escrófula es la manifestación más clásica de la presentación cervical, que es la más frecuente de la TBEP. En general los síntomas y signos pueden aparecer como crecimiento ganglionar, pérdida de peso, fiebre, sudoración nocturna, tos, por otro lado, las lesiones se pueden presentar como abscesos fríos y en ocasiones fístulas con secreción intermitente. La historia clínica generalmente es larvada con un promedio de inicio de síntomas de 3 meses hasta 1 año-11, con compromiso unilateral en el $80 \%$ y con un ganglio o conglomerado ganglionar indoloro. Incluso se reporta la ausencia de compromiso pulmonar entre el $70 \%-90 \%$ en pacientes con TBEP, lo cual puede estar dado ya que el diagnóstico de estos pacientes generalmente se realiza con confirmación anatomopatológica de tejido y no con cultivo o baciloscopía por muestra de expectoración ${ }^{12}$.

El segundo sitio en frecuencia de TBEP es la laringe con una presentación aproximada de solo el $1 \%$ de la TBC en cabeza y cuello. Clínicamente se manifiesta con estridor laríngeo, tos, odinofagia, disnea y disfagia, lo cual perfectamente puede simular una neoplasia laríngea, más si está asociado a tabaquismo y si se presenta en pacientes mayores de 60 años. El sitio de afección más frecuente son las cuerdas vocales, seguido de la epiglotis, los aritenoides, comisura posterior y espacio subglótico según lo reportado en la literatura ${ }^{13-14}$.

El diagnóstico es realizado por una combinación de manifestaciones clínicas, hallazgos de laboratorio, biopsias de tejidos y estudio de imágenes complementario. Considerando que una baciloscopía negativa, un cultivo negativo, o una biopsia no concluyente no descartan el diagnóstico de TBEP ante una sospecha clínica bien fundada, como puede ser una adenopatía abscedada con mala respuesta a antibioticoterapia y el descarte de neoplasias de la vía aereodigestiva superior.

Dentro del diagnóstico diferencial que debemos tener presente se incluyen la enfermedad linfoproliferativa o linfoma, el carcinoma escamoso de cabeza y cuello, carcinoma papilar tiroideo, procesos inflamatorios y granuloma-

\begin{tabular}{|lc|}
\hline \multicolumn{2}{|l|}{$\begin{array}{l}\text { Tabla 1. Grupos de riesgo con proporción para } \\
\text { enfermar de TBC, según Registro Nacional }\end{array}$} \\
\hline Grupos de riesgo & Proporción \\
\hline Coinfección VIH & $8,9 \%$ \\
\hline Extranjeros & $8,3 \%$ \\
\hline Alcoholismo/drogadicción & $7,3 \%$ \\
\hline Población privada de libertad & $3,6 \%$ \\
\hline Diabetes & $4,6 \%$ \\
\hline Pueblo indígena & $2,6 \%$ \\
\hline Situación de calle & $2,9 \%$ \\
\hline Contactos & $2,3 \%$ \\
\hline Personal de Salud & $0,6 \%$ \\
\hline Otras inmunosupresión & $2,2 \%$ \\
\hline
\end{tabular}

tosos como la enfermedad de Wegener, lupus eritematoso sistémico, sarcoidosis, artritis reumatoide, amiloidosis, policondritis, enfermedad de Castleman, y otras enfermedades infecciosas como sífilis, enfermedad por arañazo de gato, toxoplasmosis, actinomicosis, tularemia, e infecciones virales sistémicas como es el caso de la mononucleosis ${ }^{15}$.

\section{Técnicas bacteriológicas de diagnóstico}

La baciloscopía de esputo o expectoración es el método más sencillo, rápido, fiable y económico para el diagnóstico de los pacientes sintomáticos respiratorios. Esta técnica permite la identificación de 60\%-80\% de casos de tuberculosis pulmonar existente. Su especificidad es de $95 \%$ o más, aunque solo permite identificar bacilos acido alcohol resistente (BAAR), no diferenciando Mycobacterium tuberculosis de otras micobacterias no tuberculosas, pero su efectividad disminuye drásticamente en el estudio de TBEP.

El cultivo para aislar micobacterias es un método de diagnóstico de gran sensibilidad en pacientes bacilíferos, que permite detectar un mínimo de 100 bacilos viables por $\mathrm{ml}$ en la muestra estudiada. Esto lo diferencia de la baciloscopía que requiere una carga mayor de bacilos para resultar positiva. No obstante, en TBEP el rendimiento es pobre, ya que solo se consigue aislar Mycobacterium entre el 10\% y $69 \%$ de los casos $^{16}$, además, el resultado puede demorar entre 6 y 8 semanas con el 
consiguiente retraso en el inicio del tratamiento. Por otro lado, los métodos basados en la amplificación de ácidos nucleicos (AAN) o reacción de polimerasa en cadena (PCR) producen resultados tan rápidos como una baciloscopía, pero con mayor sensibilidad, de manera que permiten detectar casos que fueron negativos en la baciloscopia. Es útil en casos clínicos especiales en los que se necesita confirmar o descartar rápidamente la presencia del Mycobacterium tuberculosis. El método de AAN aprobado por la Organización Mundial de la Salud corresponde al Gene Xpert-RIF. Este método además de ofrecer un diagnóstico rápido de la presencia del bacilo en la muestra con cantidades de microorganismos mucho más bajas que la baciloscopía (mayor sensibilidad), tiene una alta especificidad al identificar exclusivamente a Mycobacterium tuberculosis, y además diagnostica la presencia de genes de resistencia a la rifampicina. Además, este examen es un método basado en la biología molecular que detecta en menos de dos horas la presencia de bacilos tuberculosos en diferentes fluidos orgánicos, por lo que podemos tener un resultado positivo prácticamente al terminar la cirugía de una eventual biopsia, dado que se toma una muestra de manera corriente, pudiéndose iniciar el tratamiento con la misma precocidad $^{17,18}$.

\section{Imágenes complementarias}

La radiografía de tórax puede mostrar hallazgos sugerentes de TBC en un orden de $10 \%-40 \%$ de los pacientes, sin embargo, una radiografía de tórax normal no descarta el diagnóstico ${ }^{19}$. El ultrasonido es buen método de diagnóstico en el estudio de masas cervicales, ya que, características como la forma, ecogenicidad y tamaño del ganglio o conglomerado puede orientarnos a una patología benigna o maligna, por otro lado, no existen hallazgos específicos para confirmar el diagnóstico de TBEP. Aunque el ultrasonido en combinación con la punción aspirativa con aguja fina (PAAF), puede entregarnos información con alta sensibilidad y especificidad cercana al $88 \%$ y $96 \%$ respectivamente ${ }^{20}$.

La tomografía computada en conjunto con la resonancia magnética nos puede dar información de la localización de la lesión, extensión de la enfermedad y características de los tejidos adyacentes comprometidos. Aun cuando, no existen patrones patognomónicos, una adenopatía con centro necrótico puede orientarnos ante una sospecha clínica alta, como también una adenopatía con refuerzo perimetral y calcificaciones en su interior ${ }^{21}$.

\section{Tratamiento}

El tratamiento para la TBC es bien conocido y está compuesto por una terapia antibiótica prolongada de 6 meses, dividida en un tratamiento diario por 2 meses con isonizazida, pirazinamida, etambutol y rifampicina, seguido por otra fase de cuatro meses solo con isoniazida y etambutol. Después de terminado el tratamiento de la TBEP de localización cervical, el tamaño de las adenopatías disminuye a menos de $5 \mathrm{~mm}$ en el $83 \%$ de los casos $^{22}$. La cirugía debe considerarse cuando no existe regresión de las adenopatías, fístulas o compromiso de senos paranasales. El tratamiento puede prolongarse de 6 a 9 meses si existe una pobre respuesta, se reportan tasas de recuperación de $89 \%$ a $95 \%$ con tratamientos de 6 a 9 meses respectivamente ${ }^{23}$. Algunos pacientes pueden persistir con enfermedad, o presentar recurrencia después del tratamiento.

El tratamiento para TBEP referida a la región en cabeza y cuello es idéntico, sin importar cuál sea la localización comprometida, respondiendo adecuadamente con el esquema clásico, aunque en laringe responde aún mejor con evidencia de recuperación completa en un periodo entre 2 y 6 meses.

\section{Conclusión}

El diagnóstico de tuberculosis en cabeza y cuello es desafiante dado la diversidad de las manifestaciones clínicas y en ocasiones lo poco específico de estas. La ausencia de compromiso pulmonar se observa entre el $70 \%$ y $90 \%$ y una masa cervical es el signo clínico más común. En la literatura revisada el método más utilizado en el diagnóstico es la biopsia de tejido o PAAF, siendo esta última la acción más simple y costo efectiva, sin embargo, la biopsia siempre es realizada cuando existe una alta sospecha de patología tumoral y puede evitar el retraso diagnóstico ante una PAAF no concluyente.

El estudio imagenológico nos permite iden- 
tificar el sitio exacto de infección, determinar la extensión de la enfermedad y compromiso pulmonar, entre estos se encuentra la radiografía simple, ultrasonido, tomografía computada, resonancia magnética e incluso la tomografía computada con emisión de positrones, aun cuando, ninguno de estos estudios por sí solo permite realizar el diagnóstico de certeza.

La cirugía debe considerarse ante la imposibilidad de un diagnóstico definitivo, y la necesidad de un estudio citológico a través de una biopsia excisional, teniendo en cuenta que de existir secreciones en las adenopatías se debe hacer uso de exámenes de biología molecular como el Gene-Xpert Rif si está a disposición en el centro hospitalario, para confirmar la sospecha clínica en el mismo procedimiento quirúrgico, dado la simplicidad para tomar la muestra sin procesos de por medio para su rápida evaluación en laboratorio. En relación con el tratamiento de la TBEP, este debe seguir las directrices conocidas a nivel mundial con una terapia asociada y de larga duración, la cual en Chile está a cargo de la atención primaria de salud.

Durante el año 2014 se mantiene una meseta en la velocidad de reducción de la tasa de incidencia a nivel país, lo que mantiene a Chile lejos de alcanzar la eliminación avanzada de la enfermedad y de convertirnos así en un país de baja incidencia. Existe además un aumento de la tuberculosis pulmonar con confirmación bacteriológica, lo que significa un aumento de las fuentes de contagio para la comunidad ${ }^{2}$.

Por otro lado, el fenómeno de inmigración que se ha presentado en la última década en nuestro país es un foco de potencial cuidado, dado las coberturas de vacunación de los principales países que han aportado con esta situación. Por último, la tuberculosis es una patología infecciosa poco frecuente en el ámbito de la otorrinolaringología, sin embargo, debe estar dentro del diagnóstico diferencial en el contexto de una masa cervical en estudio o una patología con pobre evolución en otro sitio anatómico de nuestra área de estudio.

\section{Bibliografía}

1. World Health Organization. Global tuberculosis report 2016 (WHO, Geneva, Switzerland, 2016).
Disponible en: http://www.who.int/tb/publications/ global_report/en/Accessed 10 Feb 2017. Consultado el: 15 de octubre de 2020.

2. MINSAL. Tuberculosis Informe de Situación Chile 2014 Programa Nacional de Control y Eliminación de la Tuberculosis Departamento de Enfermedades Transmisibles División de Prevención y Control de Enfermedades. Junio de 2015.

3. Dheda K. Barry C. Maartens G. Tuberculosis. Lancet. 2016;387:1211-1216. doi: 10.1016/S01406736(15)00151-8

4. Vaid S, LeeYY, Rawat S, Luthra A. Shah D, Ahuja AT. Tuberculosis in head and neck a forgotten differential diagnosis. Clin Radiol. 210;65:73-81. doi: 10.4103/2249-4863.161312.

5. Qian XU, Albers A, Nguyen D, Dong Y, et al. Head and neck tuberculosis: Literature review and metaanalysis. Tuberculosis. 2019;16:78-88. doi: 10.1016/j. tube.2019.04.014.

6. Mazurek GH, Jereb J, Vernon A, LoBue P, Goldberg S, Castro K, et al. Updated guidelines for using interferon gamma release assays to detect mycobacterium tuberculosis infection - United States. 2010;59(RR-5):1-25.

7. MINSAL. Manual de Organización y Procedimientos del Programa Nacional de Control y Eliminación de la Tuberculosis.

8. Departamento de Estadísticas e Información en Salud (DEIS).

9. Jha BC, Dass A, Nagarkar NM, Gupta R, Singhal S. Cervical tuberculous lympha-denopathy: changing clinical pattern and concepts in management. Postgrad Med J. 2001;77(905):185-187. doi: 10.1136/ pmj.77.905.185.

10. Fazal Iw, Habib Ur R, Ahmad I. Extrapulmonary tuberculosis in patients with cervical lymphadenopathy. J Pak Med Assoc. 2013;63(9):10947.

11. Benwill JL, Sarria JC. Laryngeal tuberculosis in the United States of America: a forgotten disease. Scand J Infect Dis. 2014;46(4):241-246. doi: $10.3109 / 00365548.2013 .877157$.

12. Sama JN, Chida N, Polan RM, Nuzzo J, Page K, Shah M. High proportion of ex- trapulmonary tuberculosis in a low prevalence setting: a retrospective cohort study. Publ Health. 2016;138:101-7. doi: 10.1016/j. puhe.2016.03.033.

13. Lim JY, Kim KM, Choi EC, Kim YH, Kim HS, Choi HS. Current clinical propensity of laryngeal tuberculosis: review of 60 cases. Eur Arch Oto-RhinoLaryngol. 2006;263(9):838-42. doi: 10.1007/s00405006-0063-5.

14. Fei B, WuZ, Min K, Zhang J, Ding C, $\mathrm{Wu} \mathrm{H}$. Interferon-gamma release assay in the diagnosis of laryngeal tuberculosis. Acta Otolaryngol. 2014;134(3):314-317. doi: 10.3109/00016489.2013.850174. 


\section{CASO CLÍNICO}

15. Rodriguez M, Aguirre F. Tuberculosis ganglionar cervical. ¿Pensamos en ella, o nos sorprende? Rev Otorrinolaringol Cir Cabeza Cuello. 2012; 72:6974.

16. Singh KK, Muralidhar M, Kumar A, Chattopadhyaya TK, Kapila K, Singh MK, et al. Comparison of in house polymerase chain reaction with conventional techniques for the detection of Mycobacterium tuberculosis DNA in granulomatous lymphadenopathy. J Clin Pathol. 2000;53:355-61. doi: 10.1136/jcp.53.5.355.

17. Vallejo P, Rodriguez JC, Searle A, Farga V. Ensayo Xpert MTB/RIF en el diagnóstico de tuberculosis. Revista chilena de enfermedades respiratorias. 2015;31(2):127-131. doi: 10.4067/S071773482015000200010 .

18. Oktay MF, Topcu I, Senyigit A, Bilici A, Arslan A, Cureoglu S, et al. Follow-up results in tuberculous cervical lymphadenitis. J Laryngol Otol. 2006;120(2):129-32. doi: 10.1017/ S002221510500277X.

19. Fontanilla JM, Barnes A, von Reyn CF. Current diagnosis and management of peripheral tuberculous lymphadenitis. Clin Infect Dis. 2011;53:555-62. doi: $10.1093 / \mathrm{cid} / \mathrm{cir} 454$.

20. Gupta A, Rahman K, Shahid M, Kumar A, Qaseem SM, Hassan SA, et al. Sonographic assessment of cervical lymphadenopathy: role of high-resolution and color Doppler imaging. Head Neck. 2011;33:297302. doi: 10.1002/hed.21448.

21. Moon WK, Han MH, Chang KH, Im JG, Kim HJ, Sung KJ, et al. CT and MR imaging of head and neck tuberculosis. Radiographics. 1997;17:391-402. doi: 10.1002/hed.21448.

22. Yuen AP, Wong SH, Tam CM, Chan SL, Wei WI, Lau SK. Prospective randomized study of thrice weekly six-month and nine-month chemotherapy for cervical tu- berculous lymphadenopathy. Otolaryngol Head Neck Surg. 1997;116(2):189-92. doi: 10.1016/ s0194-5998(97)70323-1.

23. Nalini B, Vinayak S. Tuberculosis In ear, nose, and throat practice: its presentation and diagnosis. Am J Otolaryngol. 2006;27(1):39-45. doi: 10.1016/j. amjoto.2005.07.005. 